\title{
Cloud Oriented Integrated Composite Services over SOA in Distributed Computing
}

\author{
K.Sudhakar, M.James Stephen, P.V.G.D. Prasad Reddy
}

\begin{abstract}
Service-oriented architecture (SOA) [1] is an incessant term to deal with various administrations dependent on solicitations of various clients in various ongoing applications. Still, now, a few people don't have total information about what SOA really has done, they get confounded how SOA identifies with distributed computing. On account of cloud administration usage in SOA need security mindful help creation with finegrained stream control to make sure about web administrations at execution time to share various administrations to various clients in dispersed condition? Routinely various models were acquainted with investigating secure web administrations at execution of various administrations. Because of access control infringement, they will take high execution time and other leader boundaries profoundly, and furthermore they don't control access assurance arrangements in composite administrations, which may deliver bothersome information spillage. To conquer these infringement issues in $S O A$, we present Integrated Novel Multi-Level Composite Service Model (INMLCSM) [2] to lessen infringement calculation cost dependent on customer authentic and demonstrate customer composite administrations and furthermore perform nearby/distant strategy calculation for highest customers. We acquaint idea of change factor with characterize halfway administrations. Our proposed approach portrays forceful exploratory outcomes.

Keywords: Service oriented architecture, cloud computing, access control, data flow, service oriented cloud services, service provisioning and integrating services.
\end{abstract}

\section{INTRODCUTION}

Service-Oriented Computing (SOC)[3] is the figuring perspective that utilizations benefits as head parts for making applications/game plans. Administrations are selfportraying, stage realist computational segments that help quick, negligible exertion union of appropriated applications. Administration Organizer performs limits, which can be anything from fundamental sales to tangled business structures. Administrations empower relationships to reveal their middle aptitudes consequently over the Internet (or intranet) using standard (XML-based)[4] tongues and shows and be executed by methods for a selfdepicting interface reliant on open checks. Administration structure has been extensively considered in later quite a while. Notwithstanding the way that an extensive proportion of new models and parts have been proposed, various issues in administration association actually remain unsolved.

Manuscript received on January 10, 2020.

Revised Manuscript received on January 19, 2021.

Manuscript published on February 28, 2021.

K.Sudhakar*, Department of CS\&SE, Andhra University, Visakhapatnam Email: sudhamtech@gmail.com

Dr.M.James Stephen, Professor, Department of CSE, WISTM Engineering College, Visakhapatnam Email: jamesstephenm@yahoo.com

Dr.P.V.G.D. Prasad Reddy, Senior Professor, Department of CS\&SE, Andhra University, Visakhapatnam Email: prasadreddy.vizag@gmail.com

(C) The Authors. Published by Blue Eyes Intelligence Engineering and Sciences Publication (BEIESP). This is an open access article under the CC BY-NC-ND license (http://creativecommons.org/licenses/by-nc-nd/4.0/)
Among them, get the chance to control is one of the noteworthy concerns. Regardless of the way that it is key to make get the chance to control models and strategies to telecaster part benefits and what's more composite administrations, it is in addition essential to consider when to survey the passageway control game plans. Each and every current work considers get the chance to control exactly at execution time. In any case, without the union time get the chance to control endorsement, the composite assistance may be amazingly subject to crash and burn at the execution time on account of access control encroachment, wasting creation and execution tries. To keep away from repeating failed manifestations, bookkeeping of the mistake winds up significant, achieving a more confounded what's more, monotonous piece, and execution show. To determine this issue, it is appealing to approve the passageway control approaches of individual part benefits at the advantage piece time despite the execution-time get the opportunity to control approval. Existing works don't consider association time to get the chance to control endorsement. There have been a couple of manages security-careful structure [BAR08, CAR06, DEN03, HAN06, PAC08]. These works depict security as a course of action of attributes that can be quantitatively assessed. The security properties of help can be resolved with respect to these qualities. To guarantee fundamental data resources just as administrations, the customer and master associations may portray security necessities (for example game plans[5]) for a structure with respect to these qualities. A composite help is seen as secure if the security properties of each individual assistance satisfy all the security prerequisites portrayed by the master communities and the customer. One essential issue with the above works is that they think about particularly direct characteristics, for instance, the sort of encryption estimation, such an affirmation show, etc. There are a couple of issues while considering access control at plan time. The essential issue is who should evaluate the passageway control game plans. Existing secure help structure instruments expect a totally trusted in administration author, which isn't for each situation veritable, especially in a multi-territory condition. In such a space, there are various customers in different territories and they may use unmistakable help essayists. These spread help arrangers may be in different zones from those of the web administrations. [6]A bit of this zona may have made sure about getting the chance to control plans that should not be released to a couple of gatherings (for example some help arrangers). As such, it is implausible that a help arranger is totally trusted by the providers of each and every included assistance (all the strong administrations considered by the creator, not just those truly decided) for getting to their made sure about systems.

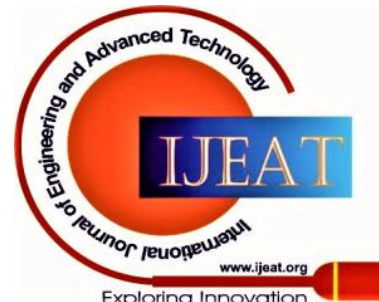




\section{Cloud Oriented Integrated Composite Services over SOA in Distributed Computing}

Along these lines, the administration essayist can't complete plan appraisal without speaking with the master associations with made sure about procedures. Essential cloud secure mindful assistance composite portrayal may show in figure 1 with various administrations

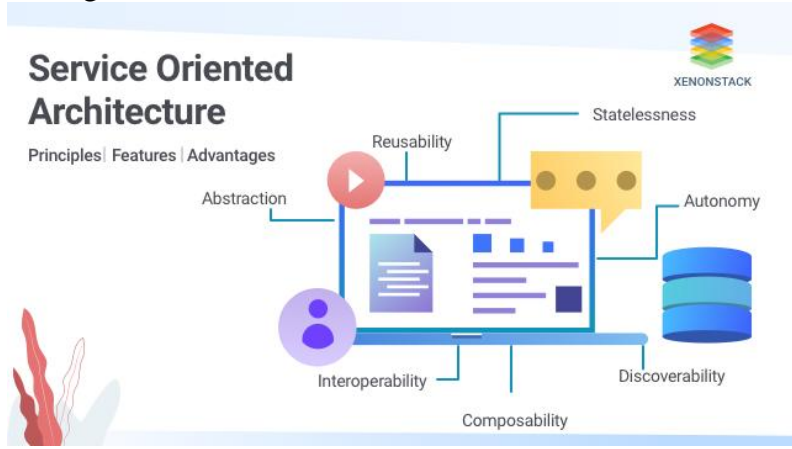

Figure 1. SOA based composite service process in Web oriented environment

We present a Integrated Novel Multi-Level Composite Service Model (INMLCSM)[7] to address the execution issue and trust in arranger issues in structure time gets the opportunity to control approval. In the chief stage, as the chase space may be broad, a more capable anyway less precise methodology is used to quickly prune the cheerful combinations. Specifically, we use the information of credible assistance piece trades to evaluate the wellbeing of candidate blends, rank them, and select the best contenders. In the subsequent stage, we consider a close by methodology appraisal system to achieve a more accurate evaluation of candidate plans. In this methodology, the essayist uses the methodologies or conceivably presentations saved or as of late downloaded from the security authorities of included administrations to locally favor the candidate manifestations. [8]To consider the execution of the proposed part, we develop a multiplication structure to imitate various shows and investigate their execution, including the three-phase piece show, the singlestage course of action show, and the show without creation time get the chance to control endorsement. The result exhibits that, without piece time get the opportunity to control endorsement, the structure and execution cost increases definitely as the accomplishment rate reduces (If the passageway control systems of section administrations are exacting, by then it is difficult to find an authentic structure and the accomplishment rate is low). [9]Exactly when the accomplishment rate is around half, even the single-stage show performs better than anything the show without structure time get the chance to control. The threephase structure show performs a lot of better than the following two instruments despite when the accomplishment rate is high (90\%). We moreover consider the execution of the shows under various help chain sizes. With the growing help chain gauge, the execution gain by the three-phase show ends up being more significant.

\section{SERVICE ORIENTED ARCHITECTURE}

SOA assurances to close the opening between present day contraptions and adventure applications. [10]The value of a model of an assistance arranged plan is the introduction of in any event one interface through a given program. These interfaces describe particular procedures that are open through the sort out. These interfaces are suggested as "administration". The people who use these administration are called advantage purchasers, and the people who give them are called pro associations. All of these administration is free, and joins the business reason and data related with promoted advantage. [11]The administration can end up interconnected with each other, as such improving the ability of the designing. Therefore, plans need to use a course of action of standards or shows to describe the message orchestrate. Those shows portray a methodology of data exchange. SOA is a term that addresses a model where the justification is isolated into different and tinier units. These units might be passed on and together shape some part of an automated business justification. A basic favored angle in the utilization of SOA is deftness in responding to changing business essentials. Interoperability and easier help transform into good position when SOA is executed precisely, regardless, if we have to execute a SOA-based plan we should consider the defending of its essential objections: the establishment must assistance flexibility, heterogeneity, passed on headway what's more, administration.

A basic piece of SOA is the division of the interface or administration (the what) from their use or substance (the how). The interface gives advantage recognizing evidence, while the substance gives business basis. Zimmermann et al. [12]suggest three degrees of pondering in SOA:

Exercises: Units of limits taking a shot at got data, having specific interfaces and returning composed responses.

- Services: Logical groupings of exercises.

- Business structures: Actions or activities to perform specific business goals by conjuring different help.

The sorts of administration offered by SOA can be named seeks after:

- Infrastructure administration: Includes security, administration and watching.

- Business-objective assistance: Includes advantage operators and cautioning, booking and work measure administration.

- Business service's: Includes administration reliant on the business basis.

According to, a SOA configuration structure may be considered as a seven-layer building.

- Layer 1: Operational systems layer; this layer joins existing structures using SOA compromise techniques.

- Layer 2: Enterprise parts layer is accountable for recognizing value and keeping up the idea of administration (QoS) of the revealed administration. [13]

- Layer 3: Services layer; in this layer, the administration decided to be consumed are found.

- Layer 4: Business structures layer, which describes the administration revealed in layer 3 .

- Layer 5: Access layer; it is made to offer start to finish responses for syntheses of administration.

- Layer 6: Integration layer; it allows the mix of different help.

- Layer 7: Management and security; it is reliable to screen, direct and take care of security.

Security in SOA Applications

Security is a champion among the most basic troubles in WSN [14].

Published By:

Blue Eyes Intelligence Engineering

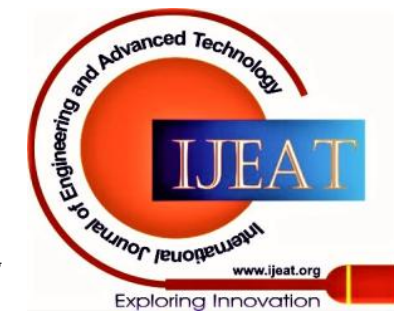

Sciences Publication

(C) Copvriaht: All riahts reserved. 
The usage of online assistance can open the eHealth to security threats, similar to some other online application. It transforms into a need that the characters of authentic customer's eHealth ought to be checked intentionally before giving access benefits.

SOA extends the amount of ways for large business systems, growing in like manner the frailty of the page, because a noteworthy number of these centers are introduced to the Internet [2-12]. Adventure level security has changed with inventive advances, and has for a very long time been tingling to keep up a "divider" between what is to be guaranteed and the people who approach on this with the purpose of balancing admittance to interlopers or unapproved personnel[15].

Not simply the plan must be protected from the people who get to the structure over the Internet, notwithstanding, it also ought to be protected from the people who approach through the intranet, and in light of the fact that inside frameworks can be gotten to from risky physical concentrations inside the affiliation. [16] The headway of the SOA use is direct comparing to security perils made by it.

\section{PROPOSED DESIGN MODEL}

In administration union, the administration author takes a reasonable help chain $<\mathrm{s} 0$, as $1, \ldots$, asn, $\mathrm{sn}+1>$ from the customer what's more, picks strong administrations to launch as $1, \ldots$, asn while satisfying the information stream control restrictions. [17]The creator at first recoups a plan of strong administrations for each hypothetical assistance asi from UDDI and produces a course of action of confident strong sytheses $\mathrm{CHO}$. For each chk in $\mathrm{CHO}$, the essayist seeks after the moved IFC oversees and affirms whether or not valid(chk.<s0, ..., sn+1>) is legitimate. The administration essayist may need to explore $\mathrm{O}(\mathrm{cn})$ candidate strong plans, where $\mathrm{n}$ is the number of hypothetical administrations in principle advantage chain and $\mathrm{c}$ is the ordinary number of candidate strong administrations per hypothetical advantage. For each candidate plan chk, the administration creator needs to affirm whether valid(chk.si, chk.sj) is substantial, for all I, j, $0>\mathrm{I}<\mathrm{n}, \mathrm{I}<\mathrm{j}>\mathrm{n}+1$. This requires creating $\mathrm{O}(\mathrm{n} 2)$ information stream control decisions. [18]The decision making may in like manner incorporate recuperating Pol(chk.si.R) from dom(chk.si).sa and chk.sj.ac from dom(chk.sj).sa. Whether or not Pol(chk.si.R) and chk.sj.ac are saved by the administration arranger, the technique evaluation may at present be exorbitant, as the administration arranger may need to survey various standards in Pol(chk.si.R) and join the results. If that Pol(chk.si.R) and furthermore a couple of characteristics in chk.sj.ac are made sure about, the administration author needs to connect with dom(chk.si).sa and also dom(chk.sj).sa with the ultimate objective to enroll auth(Attr(chk.sj),

Attr(chk.si.R), $\quad \mathrm{tf}(<\mathrm{chk} . \mathrm{si}, \quad \ldots \quad, \quad$ chk.sj-1>), Pol(chk.si.R)). If this technique evaluation measure is associated with each confident piece, the structure cost can be high.

We consider a three-phase framework to achieve capable security-careful help course of action. In the chief stage, there are various hopefuls and a gainful system is used to quickly evaluate confident concrete associations and the most promising hopefuls are picked for additional examination. [19]As opposed to truly handling valid(chk.si, chk.sj) using auth(Attr(chk.sj), Attr(chk.si.R), tf(<chk.si, .. , chk.sj-1>), Pol(chk.si.R)), the administration essayist uses the endorsement results of genuine creation trades to enlist the likelihood of valid(chk.si, chk.sj) being legitimate (shown as LL(chk.si, chk.sj)). In like way, the health assessment of each candidate sythesis chk, fit(chk), is enlisted and the best (L1 is the rate that degrees from 0 to 1 ) contenders are picked and fused into $\mathrm{CH} 1$.

In the subsequent stage, a more exact anyway possibly extra repetitive method is used to survey the rivals in CH1. For each candidate in [20]CH1, chk, the advantage author uses the saved or as of late downloaded (if the information isn't put away or is old) approaches Pol(chk.si.R) and furthermore revelations chk.sj.ac to figure auth(Attr(chk.sj), Attr(chk.si.R), $\operatorname{tf}(<$ chk.si, .., chk.sj-1>), Pol(chk.si.R)), for all I, j, $0<\mathrm{I}<\mathrm{n}, \mathrm{I}<\mathrm{j}<\mathrm{n}+1$, locally. Once in a while, the assessment of valid(chk. $<\mathrm{s} 0, \ldots$, $\mathrm{sn}+1>$ ) may be settled. If that valid(chk. $<\mathrm{s} 0, \ldots, \mathrm{sn}+1>$ ) is bogus, chk is removed from $\mathrm{CH} 1$. If valid(chk. $<\mathrm{s} 0, \ldots$, $\mathrm{sn}+1>$ ) is substantial, chk (a real association) is explicitly returned to the customer and the third stage examination is skipped. As a couple of plans and properties may be made sure about what's more, can't be downloaded, the authenticity of some contender strong structures may not be evident by the administration arranger. For this circumstance, the wellbeing regard for each candidate chk in $\mathrm{CH} 1$, fit(chk), are recomputed, and the best [21](L2 is the rate that reaches out from 0 to 1 ) candidate concrete sytheses are picked and fused into $\mathrm{CH} 2$.

In the third stage, any previously picked combinations (in $\mathrm{CH} 2$ ) should be totally affirmed. For each advantage coordinate (chk.si, chk.sj) in confident concrete piece chk in $\mathrm{CH} 2$, if Pol(chk.si.R) is made sure about, by then the advantage author needs to advance the attributes of chk.sj, Attr(chk.sj), to dom(chk.si).sa for distant procedure appraisal. If the methodology evaluation requires some made sure about characteristics of chk.sj, the administration author needs to begin an exchange meeting in which, dom(chk.si).sa recoups the guaranteed characteristics of chk.sj from dom(chk.sj).sa. In case that the third stage examination doesn't yield a plan from $\mathrm{CH} 2$, the system will rewind to the subsequent stage to pick the accompanying best $\mathrm{L} 2$ [22]candidates in $\mathrm{CH} 1$ and perform third-stage assessment again. If $\mathrm{CH} 1$ bars a considerable piece, by then the methodology will rewind to the primary stage and pick the accompanying best $\mathrm{L} 1$ cheerful pieces in $\mathrm{CH} 0$ and play out the whole strategy again. The when all is said in done three-phase creation show is given in Figure 2.

1. Run first phase analysis on $\mathrm{CH}_{0}$ and sort $\mathrm{CH}_{0}$.
2. Include top- $\mathrm{L}_{1}$ candidates in $\mathrm{CH}_{1}$.
3. Run second phase analysis on $\mathrm{CH}_{1}$ and sort $\mathrm{CH}_{1}$.
4. Include top- $\mathrm{L}_{2}$ candidates in $\mathrm{CH}_{2}$.
5. Run third phase analysis on $\mathrm{CH}_{2}$.
6. Set $\mathrm{CH}_{1}=\mathrm{CH}_{1}-\mathrm{CH}_{2}$ and go to 4 .
7. Set $\mathrm{CH}_{0}=\mathrm{CH}_{0}-\mathrm{CH}_{1}$ and go to 2.
8. Return $\varnothing$.

Figure 2. Composite service execution with different layers in service computing.

First Protocol Analysis: We develop a course of action of likelihood computation (LLC)[23] fundamentals to enlist LL(chk.si, chk.sj).

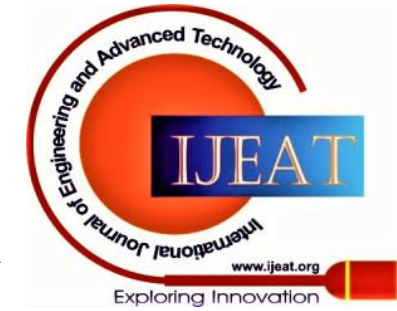




\section{Cloud Oriented Integrated Composite Services over SOA in Distributed Computing}

In any case, we consider the circumstance when there is an information stream break among chk.si and chk.sj. LLC1: If $\operatorname{tf}(<$ chk.si, .., chk.sj-1>) = NR, by then LL(chk.si, chk.sj) $=1$. Nitty gritty portrayal of first convention rendition appeared in figure 3 .

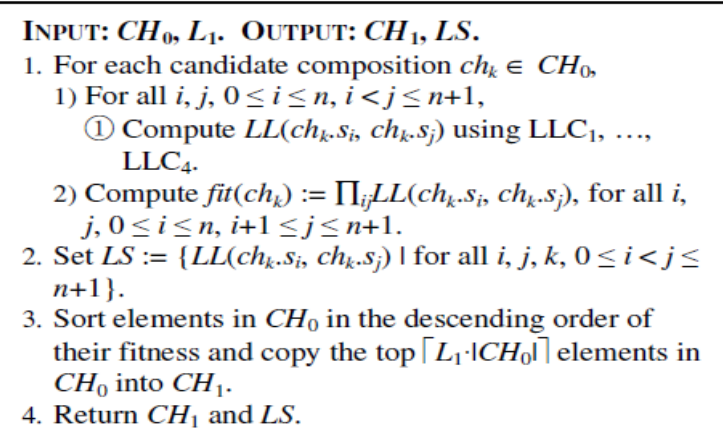

Figure 3. Different composite service processing in service computing.

If standard LLC1 isn't proper, by then we need to use the undeniable endorsement results to evaluate[24] LL (chk.si, chk.sj). To support the assessment of the likelihood, the organization author keeps up an information base VDB to store the endorsement results of all organization joins in genuine structure trades. The change factor between the two organizations furthermore impacts the information stream control decisions. Thusly, each record in VDB (for organization consolidate $(\mathrm{x}, \mathrm{y})$ ) records $(\mathrm{x}, \mathrm{y}) .<\mathrm{vresult}, \mathrm{tf}>$. Here, $(\mathrm{x}, \mathrm{y})$.vresult is the last result of valid(x, y). $(\mathrm{x}, \mathrm{y}) . \mathrm{tf}=$ $\mathrm{tf}(<\mathrm{x}, \ldots, \operatorname{pre}(\mathrm{y})>)$, where $\operatorname{pre}(\mathrm{y})$ connotes the organization not long before $\mathrm{y}$ in the primary organization chain. [25]Note that (x,y).tf is enlisted subject to the main advantage chain anyway the record doesn't need to keep the principal chain information.

We at first consider recuperating insistently planned records for (chk.si, chk.sj) to evaluate LL(chk.si, chk.sj).

Second Protocol Analysis

[26]In the subsequent stage, the organization arranger measures auth(Attr(chk.sj), Attr(chk.si.R), tf $(<$ chk.si, .., chk.sj-1>), Pol(chk.si.R)), for all I, j, $0>\mathrm{I}<$ $\mathrm{n}, \mathrm{I}<\mathrm{j}<\mathrm{n}+1$, using its put away characteristics Attr(chk.sj) and furthermore approaches Pol(chk.si.R). If the necessary information isn't for possible later use, by then the organization arranger downloads Pol(chk.si.R) from dom(chk.si).sa and chk.sj.ac (containing Attr(chk.sj)) from dom(chk.sj).sa.

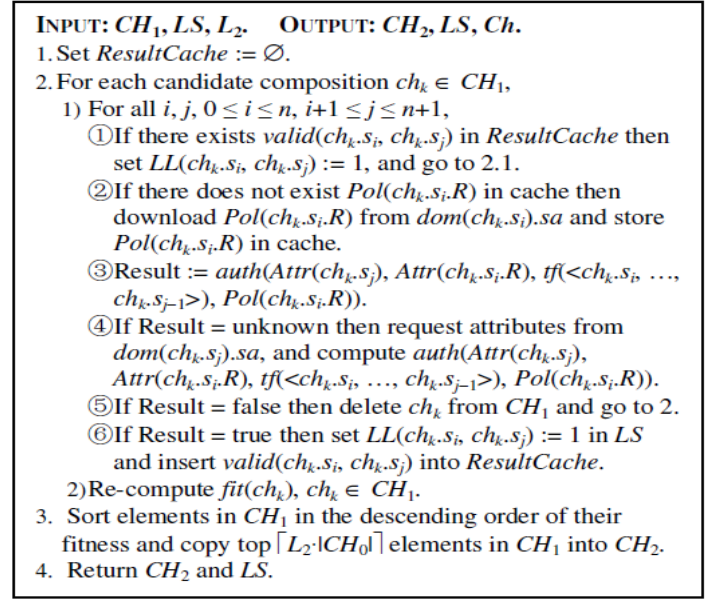

Figure 4: Composite service protocol to explore different services
To avoid possible fakes, the download of information stream control techniques and furthermore credit announcements ought to be genuinely controlled. We consider every organization author is connected with a course of action of qualities. [27]These qualities may join the name of the organization arranger, the space of the essayist, the trust level of a security master on the writer, the approval yielded to the organization arranger for downloading a couple of approaches/affirmations from a space, etc. The qualities of an organization arranger must be confirmed by a security authority. For check and non-repudiation reason, the expressed properties are joined into an attribute underwriting and set apart by the underwriter. In Definition 4.1, we describe the characteristics and property statements of organization scholars.

Definition 4.1. [28]Every organization arranger s-comp is connected with a game plan of characteristics Attr(scomp). scomp claims a game plan of attribute confirmations scomp.AC. Every trademark confirmation, scomp.ac $\in$ scomp.AC, is a validation given by a security expert to affirm that scomp holds certain characteristics scomp.ac.Attr $\subseteq \operatorname{Attr}($ scomp).

\section{Third Composite Protocol Analysis}

[28]In the third stage, the organization essayist scomp contacts the security authorities dom(chk.si).sa, for all I, $0>$ $\mathrm{I}<\mathrm{n}$, to perform distant methodology appraisal, that is, to measure auth(Attr(chk.sj), Attr(chk.si.R), $\operatorname{tf}(<$ chk.si, ... , chk.sj-1>), Pol(chk.si.R)) that have not been endorsed in the subsequent stage. In this stage, the organization creator scomp may send the saved characteristics of chk.sj, Attr(chk.sj) (fused into the trademark validation chk.sj.ac, to dom(chk.si).sa. In any case, a couple of attributes in Attr(chk.sj) may be guaranteed by dom(chk.sj).sa and can't be revealed to scomp. For this circumstance dom(chk.si).sa, for all I, $0>$ I $<\mathrm{n}$, where the evaluation of Pol(chk.si.R) requires some made sure about characteristics of chk.sj, for some $\mathrm{j}, \mathrm{I}<\mathrm{j}<\mathrm{n}+1$, need to talk with dom(chk.sj).sa to recuperate the guaranteed qualities [LEE06, OLS06].

An exchange meeting fuses a startup round, and $\mathrm{m}$ exchange adjusts. [29]The organization creator picks the maximal number of exchange adjusts $M$, and may end the course of action meeting if $\mathrm{m}$ outperforms $\mathrm{M}$. Note that, $\mathrm{m}$ may be 0 , exhibiting that all properties required by the far off methodology evaluation are starting at now given by the organization creator and, thus, no exchange is required. In the startup round, the organization arranger begins the exchange by sending the saved characteristics (consolidated into quality confirmations) required by the distant game plan appraisal inside a phenomenal message to all dom(chk.si).sa. In each round of the course of action, each security master recognizes the missing attributes required for the methodology evaluation and the security authorities who have these properties, and sends a credit request to the organization arranger. The organization arranger courses the characteristic requests to the allocated masters. [30]On getting the property request from the organization arranger, each security master evaluate its disclosure draws near and may reestablish the asked properties in a quality response. The quality response is similarly guided by the organization author

\section{Published By:}

Blue Eyes Intelligence Engineering

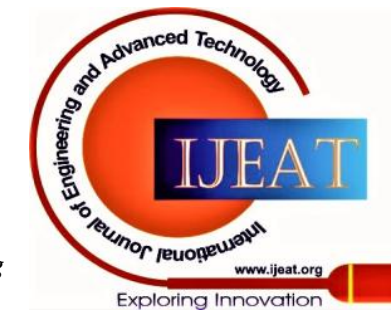


In the third stage, the organization essayist takes the best confident strong structure chk in[31] CH2 and may start exchange for the organization coordinates in chk that have not been totally affirmed. By then, valid(chk. $<\mathrm{s} 0, \ldots$, $s n+1>$ ) will have an indisputable result. If the result is legitimate, chk is returned to the customer. In case the result is bogus, chk is ousted from $\mathrm{CH} 2$ and the accompanying most shocking situated confident creation will be picked to experience a comparative endorsement measure. The third stage show is showed up in Figure 5

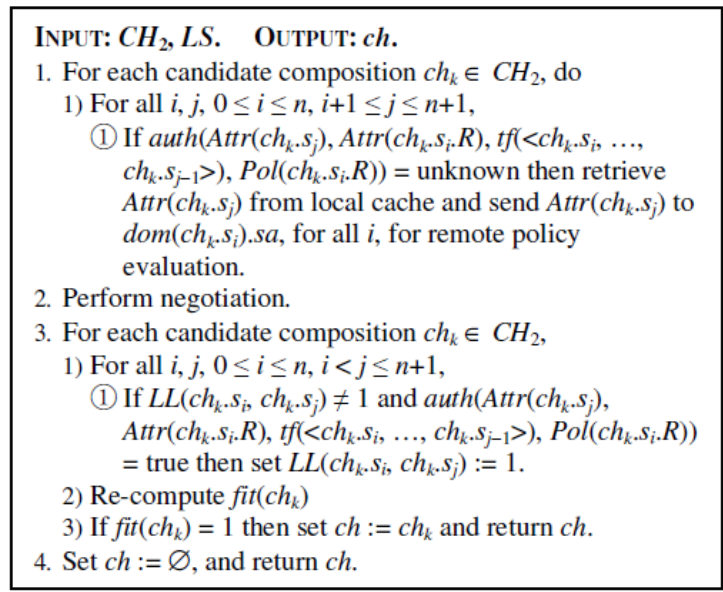

\section{Figure 5. Secure Composite service to analyze third} protocol representation.

In this paper, we consider a synchronized game plan show in which, the organization creator goes about as the course of action delegate between all security specialists[32]. This is to avoid direct exchange between all dom(chk.si).sa, $0>$ I $<$ n, and all dom(chk.sj).sa, $\mathrm{I}<\mathrm{j}>\mathrm{n}+1$, which results in $\mathrm{O}(\mathrm{n} 2)$ plan channels. In the midst of the plan, the organization essayist revises the groups got from different security specialists, and consolidations the packs with a similar objective into one message. This strategy can diminish the amount of game plan channels into $O(n)$. [33]Note that made sure about qualities should be mixed in the midst of correspondence. As such, disregarding the way that the message trade is through the organization arranger, the organization essayist doesn't have the foggiest idea about the certified quality characteristics.

Prior to the completion of the exchange meeting, either all dom(chk.si).sa recuperates the necessary characteristics for game plan appraisal, or the exchange misses the mark. [34]For the last case, the advantage author will set $\operatorname{valid}(\mathrm{chk} .<\mathrm{s} 0, \ldots, \mathrm{sn}+1>)$ to bogus. For the past case, auth(Attr(chk.sj), Attr(chk.si.R), $\mathrm{tf}(<\mathrm{chk} . \mathrm{si}, \ldots$, chk.sj-1>), Pol(chk.si.R)), for all I, j, $0>$ I $<$ n, I $<$ j $<$ n +1 , will be either substantial or bogus and, consequently, valid(chk. $<\mathrm{s} 0, \ldots, \mathrm{sn}+1>$ ) can be totally picked.

\section{EXPERIMENTS}

In this part, we characterize the trial arrangement of our proposed way to deal with characterize security mindful composite help with customary composite assistance conventions i.e (P1) [35]three-phase convention pecking order, (P2) single assistance stage convention, and composite time access control convention (P3) under execution achievement rates administration handling lengths with various variations L1 and L2 (composite help estimated boundaries).

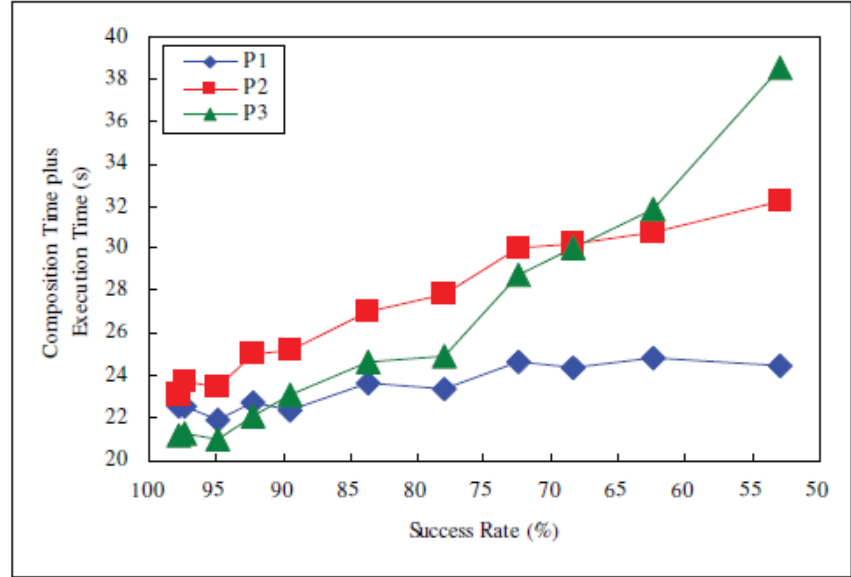

Figure 6. Performance of composite services processing with success rate and time.

In Figure 6, we consider the execution of P1, P2, and P3 under different accomplishment rates. As can be seen, when the accomplishment rate reduces, the time required by $\mathrm{P} 3$ augments radically since the wasted execution tries ended up being more extraordinary. [36]Right when the accomplishment rate is underneath $68 \%$, the cost of P3 is higher than the single-stage show (P2). (Note that in P1 and $\mathrm{P} 2$, we need to perform get the opportunity to control endorsement at plan time, and re-attempt it at execution time to ensure security). The execution addition of P1 ends up basic even at a high accomplishment rate. Right when the accomplishment rate is $97 \%$, P1 is simply $3 \%$ faster than P2 and performs possibly more horrible than P3. Exactly when the accomplishment rate is 53\%, P1 is $24 \%$ snappier than P2 and $37 \%$ speedier than P3.

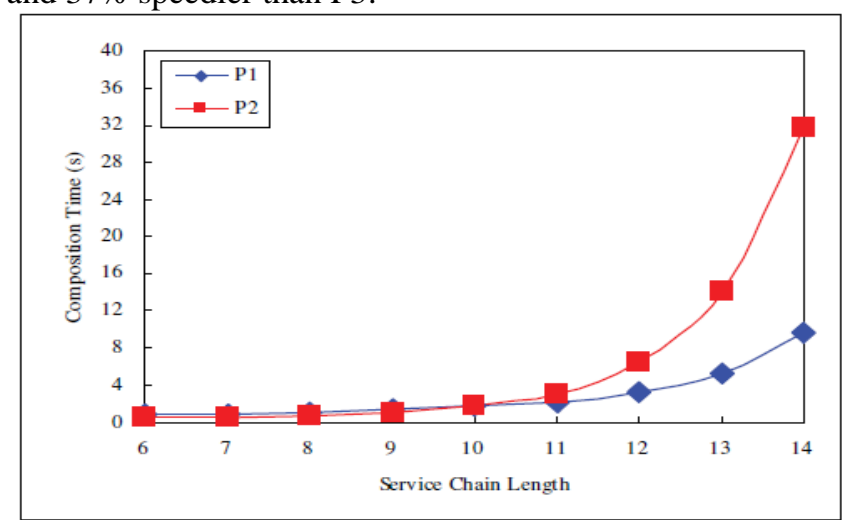

Figure 7. Performance of composite service representative service execution.

In Figure 7, we investigate the association season of P1 and P2 under different organization chain lengths at a by and large high accomplishment rate (running from 88\% (length $=6$ ) to $74 \%$ (length $=14)$ ).[36]For shorter organization chains (length $>10$ ), the two strategies can find a significant association quickly. Sometimes, the threephase show (P1) may even play out to some degree more lamentable than P2 due to the extra time spent for health check. For longer organization chains (length $>10$ ), P1 performs a lot of better than P2, from 30\% upgrade at last[37] 11 to $70 \%$ improvement at long last 14 . From the creating design, the execution upgrade in the three-phase plan can be significantly more basic with extending issue measure.
Blue Eyes Intelligence Engineering and Sciences Publication (c) Copyright: All rights reserved.

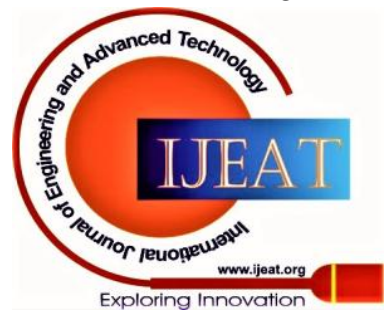




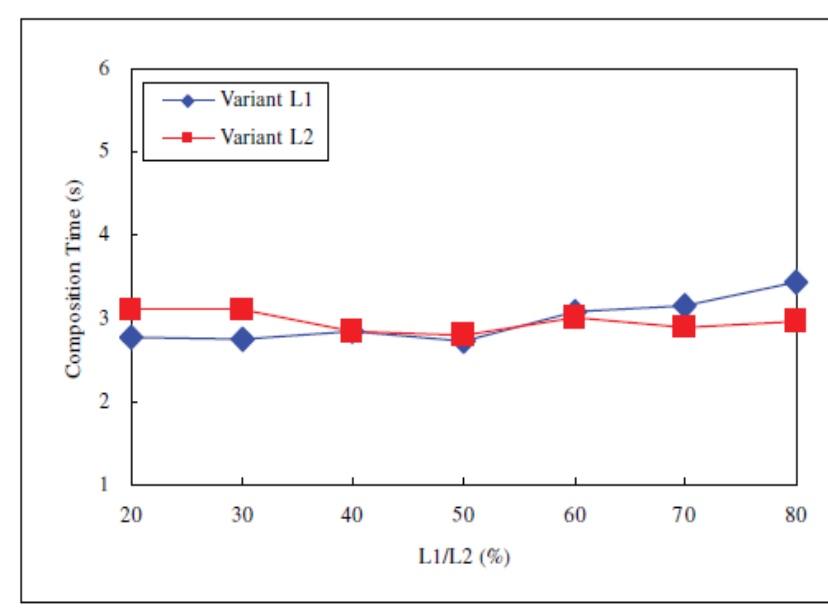

Figure 8. Performance of service utilization in service composite services.

Figures 8 show how the decision of best cheerful extents in the first and second stages, L1 and L2, can influence the execution of the three-phase structure show. [38]The result exhibits that L1 and L2 don't on a very basic level influence the execution. With different L1, the course of action season of the three-phase show influences some place in the scope of $2.3 \mathrm{sec}$ and 3sec. With different L2, the thesis time influences some place in the scope of $2.6 \mathrm{sec}$ and 2.8sec.

\section{CONCLUSIONS}

In this paper, we propose and implement an innovative Novel Multi-Level Information Integrated Protocol (NMLIIP) [39]to reduce violation computation cost based on client historical and prove client composite services. And this approach also gives better composite time intervals data flow process in between server to different client present in business organizations. Our proposed approach reduces execution of different composite services with information control violations. Using this approach, we can quickly update and eliminate invalid or unauthorized data flow control services. Our experimental results show efficient performance of proposed approach with different composite services processing and deriving services in distributed environment.

\section{REFERENCES}

1. Wei She, I-Ling Yen, Bhavani Thuraisingham, "Security-aware Service Composition withFine-grained Information Flow Control", IEEE TRANSACTIONS ON SERVICES COMPUTING, 2015.

2. Ardagna, C.A., Vimercati, S.D.C.D., Paraboschi, S., Pedrini, E., Samarati, P., Verdicchio, M., "Expressive and deployable access control in open web service applications," IEEE Transactions on Services Computing, vol. 4, no. 2, pp. 96-109, 2011.

3. Bartoletti, M., Degano, P., Ferrari, G.L., Zunino, R., "Semanticsbased design for secure web services," IEEE Transactions on Software Engineering, vol. 34, no. 1, pp. 33-49, 2008.

4. Bhatti, R., Bertino, E., Ghafoor, A., "A trust-based context-aware access control model for web-services," IEEE International

5. Carminati, B., Ferrari, E., Hung, P.C.K., "Security conscious web service composition," IEEE International Conference on Web Services, pp. 489-496, 2006.

6. Denker, G., Kagal, L., Finin, T., Paolucci, M., Sycara, K., "Security for DAML web services: annotation and matchmaking," Lecture Notes in Compute Science, vol. 2870/2003, pp. 335-350, 2003.

7. Jun Han, Kowalczyk, R., Khan, K.M., "Security-oriented service composition and evolution," Asia Pacific Conference on Software Engineering, pp. 71-78, 2006. Conference on Web Services, pp. 184-191, 2004.

8. Warschofsky, R., "A web service architecture for decentralized identity- and attribute-based access control," IEEE International Conference on Web Services, pp. 551-558, 2009.

9. Syed Mohtashim Abbas Bokhari, Umm-e-Habiba, Farooque Azam, and Muhammad Abbas, "Limitations of Service Oriented Architecture and its Combination with Cloud Computing", Bahria University Journal of Information \& Communication Technologies Vol. 8, Issue 1, April 2015.

10. D. Krafzig, K. Banke and D. Slama. "Enterprise SOA Serviceoriented architecture best practices", Prentice Hall Professional Technical Reference, Indianapolis IN, pp. 57, 2005

11. I. Cartright and E Doemenburg, "Time to jump on the bandwagon in IT", British Computer Society, UK, 2006

12. E. Knorr and O. Rist, "10 steps to SOA in Info World, SanMateo", vol. 27, no. 45, 2005

13. H. J. Scholl and R. Klischewski, "E-Government Integration and Interoperability: Framing the Research Agenda", International Journal of Public Administration, vol. 30, no. 8-9, pp. 889-920,2007.

14. K. H. Bennett, V. T. Rajlich and N. Wilde, "Software Evolution and the Staged Model of the Software Lifecycle", Advances in Computers, Volume 56, Academic Press, pp. 1 - 54, 2002.

15. Gaoyun Chen, Jun Lu and Jian Huang, Zexu Wu, "SaaAS - The Mobile Agent based Service for Cloud Computing in Internet Environment, Sixth International Conference on Natura Computation, ICNC 2010, pp. 2935-2939, IEEE, Yantai, Shandong, China, ISBN: 978-1-4244-5958-2, 2010.

16. W. De Pauw, M. Lei, E. Pring, L. Villard, M. Arnold and J. F. Morar, "Web Services Navigator: Visualizing the execution of Web Services", IBM Systems Journal, vol. 44, no. 4, pp. 821-845, 2005.

17. S. Halle, T. Bultan, G. Hughes, M. Alkhalaf and R. Villemaire, "Runtime Verification of Web Service Interface Contracts", Computer, vol. 43, no. 3, pp. 59-66, 2010

18. J. Coffey, L. White, N. Wilde and S. Simmons, Locating Software Features in a SOA Composite Application, Eighth IEEE European Conference on Web Services, ECOWS'10, pp. 99-106, 2010.

19. Various Algorithms \& Techniques Driving Data Science for Big Data, by K.Sudhakar, in International Journal of Innovative Technology and Exploring Engineering (IJITEE) ISSN: 2278-3075, Volume-9 Issue-5, March 2020

20. Enormous Information Examination using Big Data in a Distributed Environment with Profound Learning of Next Generation Interruption Identification Framework Enhancement by K.Sudhakar in International Journal of Innovative Technology and Exploring Engineering (IJITEE) ISSN：2278-3075, Volume-9 Issue-1, November 2019

21. An Effective Security Verification Model for Big Data by using potent key length for real time systems by K. Sudhakar in International Journal of Recent Technology and Engineering (IJRTE) ISSN: 2277-3878, Volume-7, Issue-6S4, April 2019

22. Estimation of Erosion and Deposition of Krishna River Bank using Remote Sensing \& GIS by K.Sudhakar, International Journal of Recent Technology and Engineering (IJRTE) ISSN: 2277-3878, Volume-7, Issue-6S, March 2019

23. The Effect of Connected Things with Intelligent Management Platform- An IoT Solution (A Research Study on Nokia) by Mr.K.Sudhakar, Mr.S.Babu Rajendra Prasad. In International Journal of Scientific \& Engineering Research Volume 10, Issue 5, May-2019 ISSN 2229-5518

24. Privacy preserved data transmission in any cloud for Big Data files using MapReduce with Security using AES Algorithm and Stegnography by K.Sudhakar,Lakshmi Saroja Ponnapalli, Tullimilli Jayasree Kalyani, Vandrasi Komal Kumar, Tunuguntla Tarun Venkata sai Ramakrishna in ALOCHANACHAKRA, ISSN:22313990, Vol9/Issue 6 June 2020.

25. An Accident / Incident Reporting Device using IoT K.Sudhakar, K.Sunayana, G.Sravya, K.Priyanka at International Conference on Contemporary Engineering \& Technology -ICCET2018.

26. PSCMR Polytechnic Website using Open Source Wordpress Tool K.Sudhakar, T.N.V.D.C Bidisha, Md.Nawaz, Sk.Reshma International Conference on Contemporary Engineering \& Technology-ICCET-2018

27. Scale-Out Cybersecurity Investigation Based On Moderate, IndustryStandard Framework K.Sudhakar, B.Hanumantha Rao, International Journal of Advances in Arts, Sciences and Engineering 3 (7), 31-34 2015

\section{Published By:}

Blue Eyes Intelligence Engineering

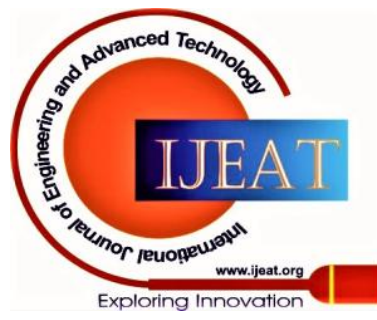


28. Emerging issues in corporate governance: an international review by considering the financial markets, regulatory environment and IT,K.Sudhakar, U Chandramouli in International journal of business, management and allied sciences. ISSN:23494638.Vol2.Issue3.(IJBMAS.IN)2015

29. An Android Application for Online Library Management System K Sudhakar International Journal of Computer Science \& Mechatronics(IJCSM.IN) 3 (1) 2017

30. Empowering of Internet of Things to Solve the Customer Engagement Paradox by K.Sudhakar in International Journal of Scientific \& Engineering Research, Volume 7, Issue 7, July-2016 20ISSN 22295518

31. IoT Routing classification and protocols with Autonomous Systems of Things K.Sudhakar, S.Rajendra Babu, B.SarathChandra First National Conference on Recent Trends in Advanced Computing 1 (Special 2016)

32. Best Practices in Vulnerability Management: "The EiQ Networks' SOCVue Vulnerability, Management" Administration Perspective VPG K.Sudhakar, G.Rambabu International Journal of Research Computer Science \&Engineering 1 (Special2016)

33. Refining Access Points and Web browsers KS Mr.Vamsi Krishna International Journal of Computer Science \& Mechatronics(OnlineISSN:2455)2015

34. Synthesizing Digital-to-Analog Converters and Internet QoS Using Hipe KS G.Madhusudana Rao International Journal of Computer Science \& Mechatronics(Online:ISSN:2455)2015

35. Decoupling \& Journaling File Systems from Consistent Hashing in SMPs KR K.Sudhakar International Journal of Computer Science \& Mechatronics 1 (1)2015

36. A case study on high performance, seamless scale for mercadolibre's openstack private cloud for scale-out, performance and inline efficiency K.Sudhakar, S.Krishna Kishore International Journal of Engineering Research-Online (ISSN: 2321-7758)3(4)- 2015

37. Hadoop- a modern data architecture for accelerating earnings growth in banking and insurance KS VSRK.Prasad.G at International Journal of Engineering Research-Online (ISSN: 2321-7758)

38. The impact of api-change in android applications and its implications in user ratings K.Sudhakar, B.SarathChandra at International Journal of Engineering Research-Online(ISSN: 2321-7758)

39. Enhancement of cost savings using virtualization in it industry is a bench mark for cloud technology SKK K.Sudhakar International Journal of Engineering Research-Online 3(4), 261-263/ 2015

\section{AUTHORS: PROFILE}

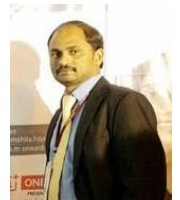

K.Sudhakar, PhD Scholar of Computer Science and Systems Engineering at Andhra University, Visakhapatnam, with research interests in Cloud Computing services, distributed computing, Big Data and Parallel Computing

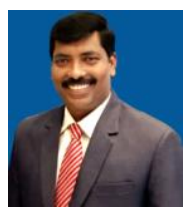

Dr.M.James Stephen is Professor of Computer Science and Principal at WISTM Engineering College, Visakhapatnam. Over two decades of Teaching and research experience Professor James is a visiting professor for various International Universities with research interests in Artificial Intelligence, Image Processing and Cloud Computing.

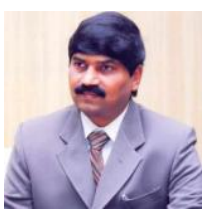

Prof PVGD Prasad Reddy is Senior Professor of Computer Science and Systems Engineering from Andhra University, with research areas of Wireless Networks, IoT, Security, Cloud Computing and Software Engineering. He is the emeritus professor for various National and International Universities and Visakhapatnam
Honorable Vice Chancellor to Andhra University,

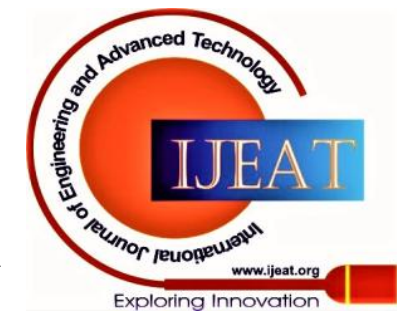

